\title{
structures des réseaux poreux et propriétés pétrophysiques des roches
}

\section{structures of porous networks and petrophysical properties of rocks}

\author{
Ch. JACQUIN \\ Institut Français du Pétrole
}

Rev. Franç. Géotech. n० 49, pp. 25-42 (octobre 1989)

\section{Résumé}

On illustre par des exemples l'existence de structures compliquées dans les matériaux géologiques et on présente quelques résultats.

En appliquant des techniques simples (variogramme, analyse fractale), il est possible d'estimer les échelles de longueur caractéristiques correspondant à ces structures et de discuter la validité de la notion de "Volume Elémentaire Représentatif $"$.

En conclusion, on pose le problème de la validité des mesures pétrophysiques conventionnelles et on donne quelques orientations pour des recherches complémentaires, prenant en compte la complexité des matériaux géologiques.

\section{Abstract}

This paper illustrates the complicated structures of geological materials and presents some results.

Applying simple techniques (variogram, fractal analysis) it is possible to estimate the characteristic length scales corresponding to these structures and to discuss the validity of the concept of "Elementary Representative Volume".

The conclusion examines the validity of conventional petrophysical measurements, and gives orientations for future research work, taking in account the complexity of geological materials. 


\section{INTRODUCTION : LES PROBLÈMES PRATIQUES}

\subsection{Grandeurs pétrophysiques et échantillons « représentatifs »}

Bien que leur définition apparaisse formellement simple, les grandeurs pétrophysiques qu'utilise l'ingénieur posent un problème pratique : celui de leur mesure.

La difficulté apparaît même pour la notion la plus élémentaire, celle de porosité.

On définit, apparemment sans ambiguïté,

$\varnothing$ : porosité

$$
\varnothing=\frac{V_{\mathrm{V}}}{\mathrm{V}_{\mathrm{T}}}
$$

$V_{V}$ : volume des pores

$\mathrm{V}_{\mathrm{T}}$ : volume total.

En fait, cette écriture même traduit le caractère discontinu du milieu (coexistence de pores et grains solides) et l'on conçoit que la notion de porosité n'ait de signification que si le volume total de l'échantillon est suffisant, autrement dit si l'échantillon est « représentatif $x$.

\subsection{Notion de «volume élémentaire représentatif »}

Ce n'est qu'à une échelle suffisante pour que les pores soient statistiquement confondus que la notion de porosité en tant que " propriété matricielle » prend une signification et que sa mesure devient utilisable.

Cette échelle minimum définit ce que l'on appelle classiquement le « volume élémentaire représentatif " ou $\propto$ volume d'homogénéisation $*$, correspondant au plus petit échantillon représentatif.

\subsection{Quelques points d'interrogation}

Trois problèmes se posent alors:

1. Comment estimer cette échelle caractéristique, audelà de laquelle les notions et les lois pétrophysiques classiques (relatives à un milieu homogène équivalent au milieu réel) sont utilisables?

2. Est-il possible de tirer parti d'informations obtenues à des échelles inférieures à la taille minimum correspondant au "volume élémentaire représentatif " et d'établir par exemple des lois physiques prenant en compte le caractère non homogène ou « irrégulier " que possède le matériau considéré à petite échelle? 3. N'existe-t-il, pour un matériau donné, qu'une seule échelle caractéristique, autrement dit dans quelles limites l'extrapolation au-delà du volume élémentaire représentatif peut-elle être réalisée?

On tentera, dans ce qui suit, d'apporter quelques éléments de réponse à ces questions en examinant successivement :
- les outils (ou techniques) qui permettent d'aborder les problèmes évoqués ;

- les résultats obtenus en appliquant ces outils à l'analyse de certaines grandeurs pétrophysiques auxquelles on fait appel pour décrire notamment les écoulements et les déplacements de fluides dans les roches poreuses.

\section{LES OUTILS}

\subsection{Les techniques fractales}

\subsubsection{Notions sur les objets fractals}

Un exemple simple et classique de ligne possédant un caractère fractal est la courbe de Von Koch, obtenue comme suit (fig. 1) :

- Stade initial

- On part d'un segment de droite de longueur $l_{0}$.

- Stade 1

- On remplace le tiers central du segment par les deux autres côtés du triangle équilatéral dont le troisième côté est ce tiers central.

- Stade 2

- On renouvelle l'opération sur chacun des quatre segments constituant la ligne obtenue à l'étape précédente et ainsi de suite.

Estimons la longueur L de cette ligne avec un étalon de mesure de longueur I qui caractériste le degré de finesse d'observation accessible (c'est-à-dire que les détails dont la taille est inférieure à 1 échappent à l'observation).

- Pour $1=1_{0}$, la courbe se confond avec le segment initial. On a $L=10$

- Pour $1=1_{0} / 3$, la courbe se confond avec les quatre segments du stade de construction $n^{\circ} 1$, et on $\mathrm{a} L=4 \frac{\mathrm{l}_{0}}{3}$.

- Pour $1=\frac{1_{0}}{9}$ on aura de même $\mathrm{L}=16 \frac{1_{0}}{9}$.

- Pour $1=\frac{1_{0}}{3^{\alpha}}(\alpha$ entier $)$, on distingue les détails obtenus au stade de construction $\alpha$ et on a $L=4^{\alpha} \frac{l_{0}}{3^{\alpha}}$

La loi générale d'évolution de $\mathrm{L}$ en fonction de 1 s'obtient en éliminant $\alpha$ entre 1 et L.

On obtient :

$\log L=\log 1(1-D)+C$ st 


$$
D^{f}=\frac{\log 4}{\log 3}
$$
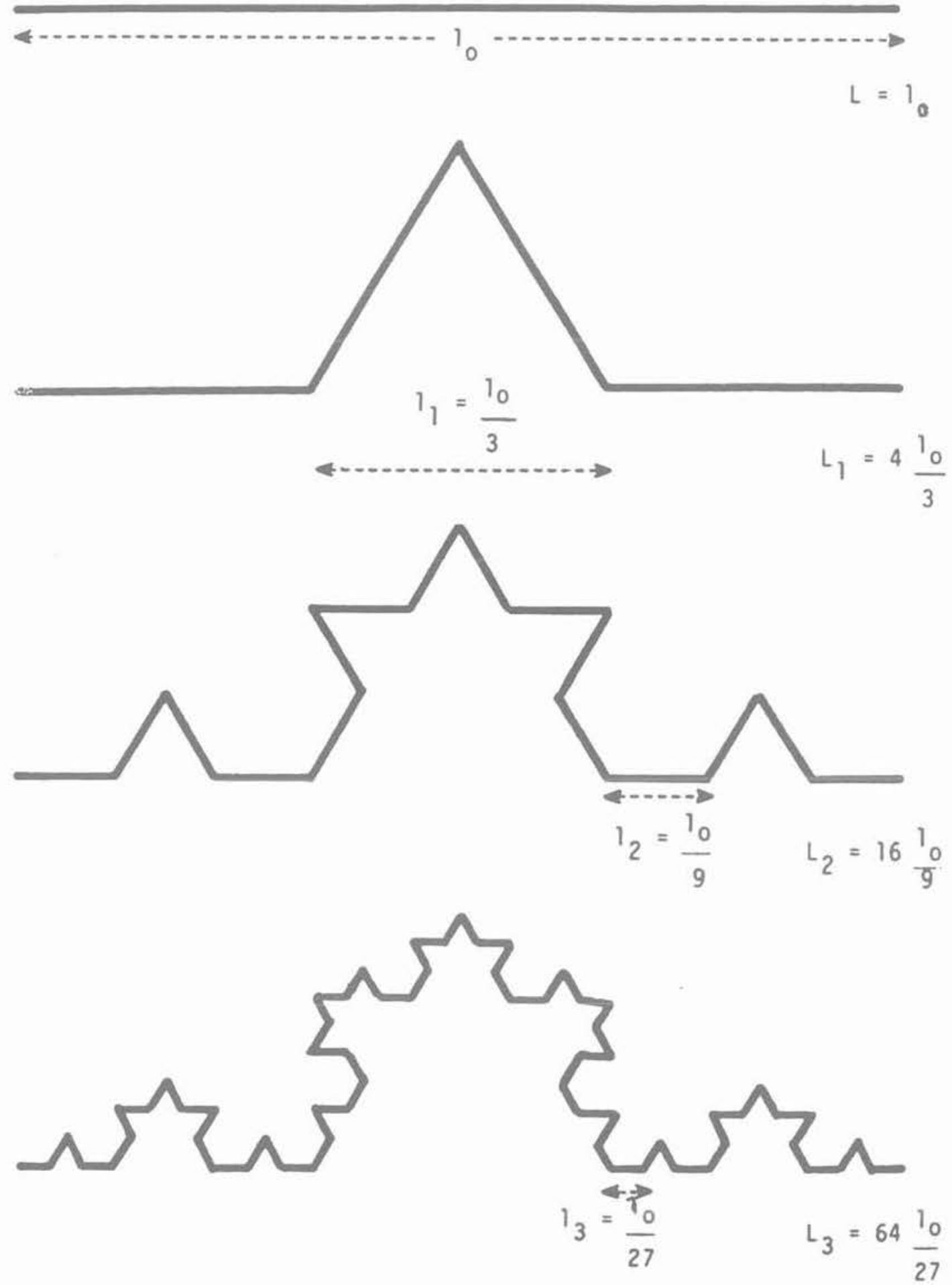

Fig. 1. - Courbe de Von Kock.

Fig. 1. - Von Koch's curve. 
avec $D=$ dimension fractale

$$
\text { ici } D=\frac{\log 4}{\log 3}=1,26
$$

On voit que, en coordonnées bilogarithmiques, $\mathrm{L}=$ $\mathrm{f}(1)$ est représentée par une droite dont la pente est 1 - D.

En résumé :

a. Le terme d'objet fractal est de création récente (MANDELBROT, vers 1965), mais des exemples théoriques ou pratiques de tels objets ont été signalés depuis longtemps (la construction de la courbe de VON KOCH a été décrite par celui-ci en 1904).

b. En se plaçant, pour simplifier, dans l'espace à deux dimensions (plan) et en reprenant l'exemple de la courbe de $\mathrm{VON} \mathrm{KOCH}$, on peut résumer les caractéristiques des objets fractals.

Si l'on poursuit la construction jusqu'à l'infini, on obtient une courbe qui présente des propriétés particulières :

- la mesure de sa longueur $\mathrm{L}$ dépend du pas de mesure 1 et augmente indéfiniment quand $1-0$;

- la loi de variation de $\mathrm{L}$ en fonction de I est du type : $\mathrm{L} \alpha \mathrm{I}^{1-D}$ avec $\mathrm{D}=$ dimension fractale, comprise entre 1 et 2 ;

- la courbe présente une homothétie interne, c'està-dire que si on l'observe à des grossissements de plus en plus élevés, en gardant le même champ d'observation, limage reste toujours la même.

\subsection{Le variogramme}

Le variogramme est l'outil de base pour l'étude des problèmes posés par les grandeurs mesurées dans la nature (teneur en minerai, puissance d'une formation, perméabilité, etc.) et que l'on considère dans le cadre de la théorie des Variables Régionalisées (MATHERON, [38]).

Ces grandeurs présentent en effet un double aspect: - un aspect aléatoire traduisant le fait que les valeurs numériques peuvent varier considérablement d'un point à un autre de l'espace ;

- un aspect régionalisé : qui interdit de regarder les valeurs numériques comme indépendantes et interchangeables dans l'espace. L'analyse par des méthodes statistiques habituelles est alors inadaptée.

La théorie des Variables Régionalisées permet:

- d'exprimer ces caractéristiques structurales sous une forme mathématique adéquate ;

- de résoudre le problème de l'estimation d'une Variable Régionalisée à partir d'un échantillon fragmentaire.

Désignons par $\mathrm{x}$ un point de l'espace exploré et par $\mathrm{f}(\mathrm{x})$ la valeur prise en ce point par la Variable Régionalisée considérée. Le variogramme peut être défini comme exprimant la variance des accroissements de $f(x)$; il a pour expression :

$$
\gamma(\vec{h})=\frac{1}{2} E\left[\left\{f\left(x_{0}+\vec{h}\right)-f\left(x_{0}\right)\right\}^{2}\right]
$$

C'est donc une fonction de la distance $\mathrm{h}$ séparant les points $\mathrm{x}_{0}$ et $\mathrm{x}_{0}+\mathrm{h}$; le symbole $\mathrm{E}$ [ ] représente l'espérance mathématique de l'expression entre crochets ; $\gamma(\mathrm{h})$ est donc, pour h fixé, la moyenne de cette quantité lorsque le couple de points $\mathrm{x}_{0}$ et $\mathrm{x}_{0}+$ $\mathrm{h}$ se déplace de manière à occuper toutes les positions possibles de l'objet étudié

On verra, sur les exemples présentés plus loin, que le variogramme traduit les traits structuraux de la variable $f(x)$.

\section{QUELQUES RÉSULTATS}

\subsection{Analyse fractale de sections planes de réseaux poreux}

1. La figure 2 représente l'image d'une lame mince de Grès de Fontainebleau, matériau naturel particulièrement simple et bien étudié.

La porosité, de type strictement intergranulaire, apparaît nettement sur cette image.

2. Les figures 3 et 4 fournissent deux exemples de sections planes de roches carbonatées poreuses. Ces images ont été obtenues à partir de lames minces de calcaire dolomitisé.

3. Pour ces deux types de roches, l'analyse géométrique du réseau poreux semble donc tout à fait réalisable ; les interfaces pores-minéral sont nets et bien dessinés, et les échelles de longueur caractéristiques apparaissent accessibles à la mesure sur ces images, étant donné les grandissements utilisés. Le seuil de coupure inférieure que l'on est bien obligé d'accepter, et qui introduit une dimension minimale en deça de laquelle les détails deviennent inobservables, reste petit vis-à-vis de l'objet.

Tel ne serait pas le cas si l'on cherchait à appliquer ce mode d'analyse à certains autres types de porosité (exemple: porosités inter et intra-granulaire de calcaires oolitiques).

4. L'analyse de ces images est réalisée de manière à mettre en évidence leur caractère fractal éventuel ; cette particularité de la structure géométrique sera confirmée ou infirmée a posteriori par l'examen des résultats.

Suivant la nature de l'objet étudié, on procède de deux manières :

- pour l'analyse d'un pore isolé (fig. 3), on retient comme information de départ son contour, c'est-à-dire 


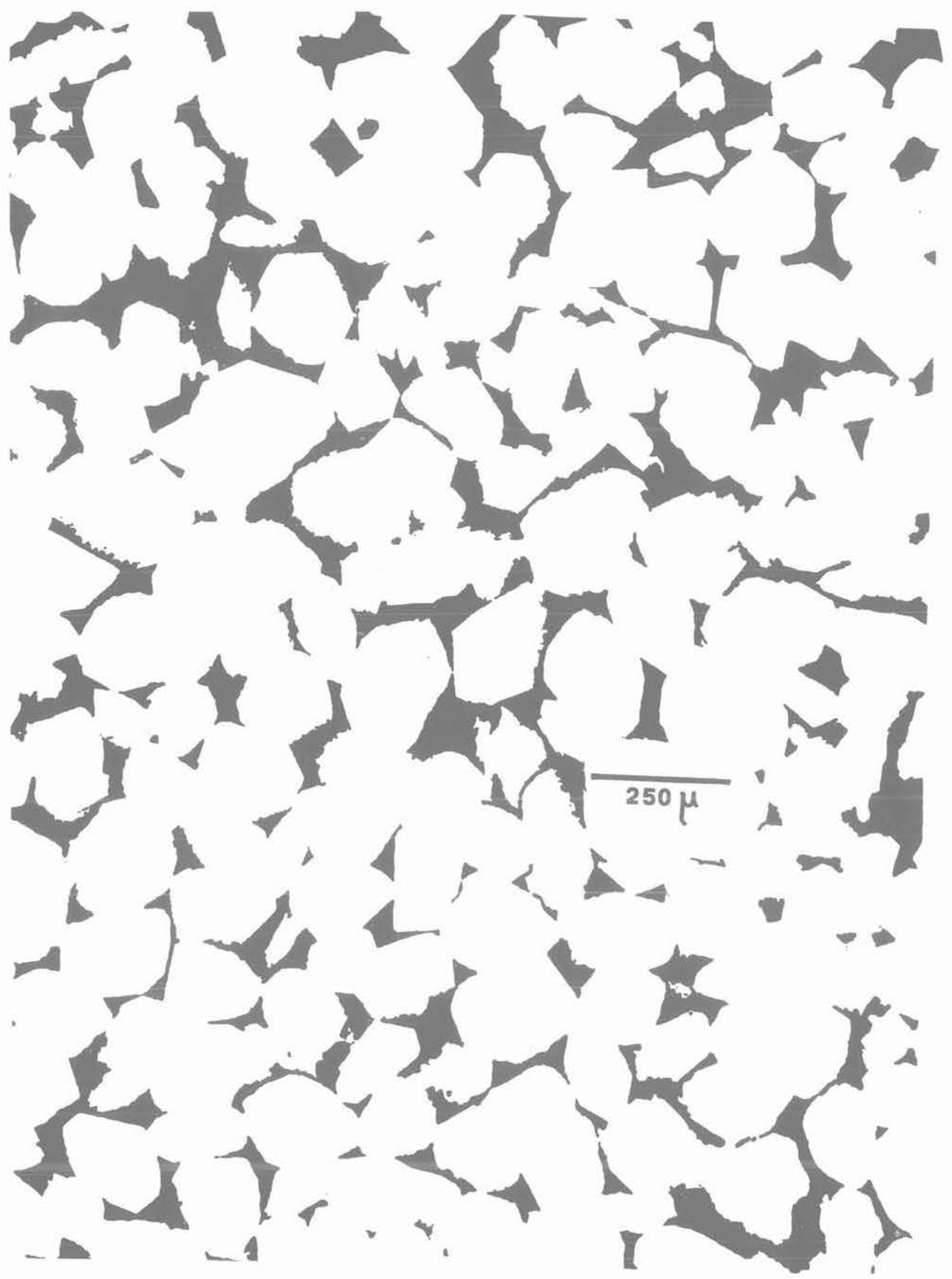

Fig. 2. - Lame mince. Grès de Fontainebleau.

Fig 2. - Thin section. Fontainebleau sandstone. 


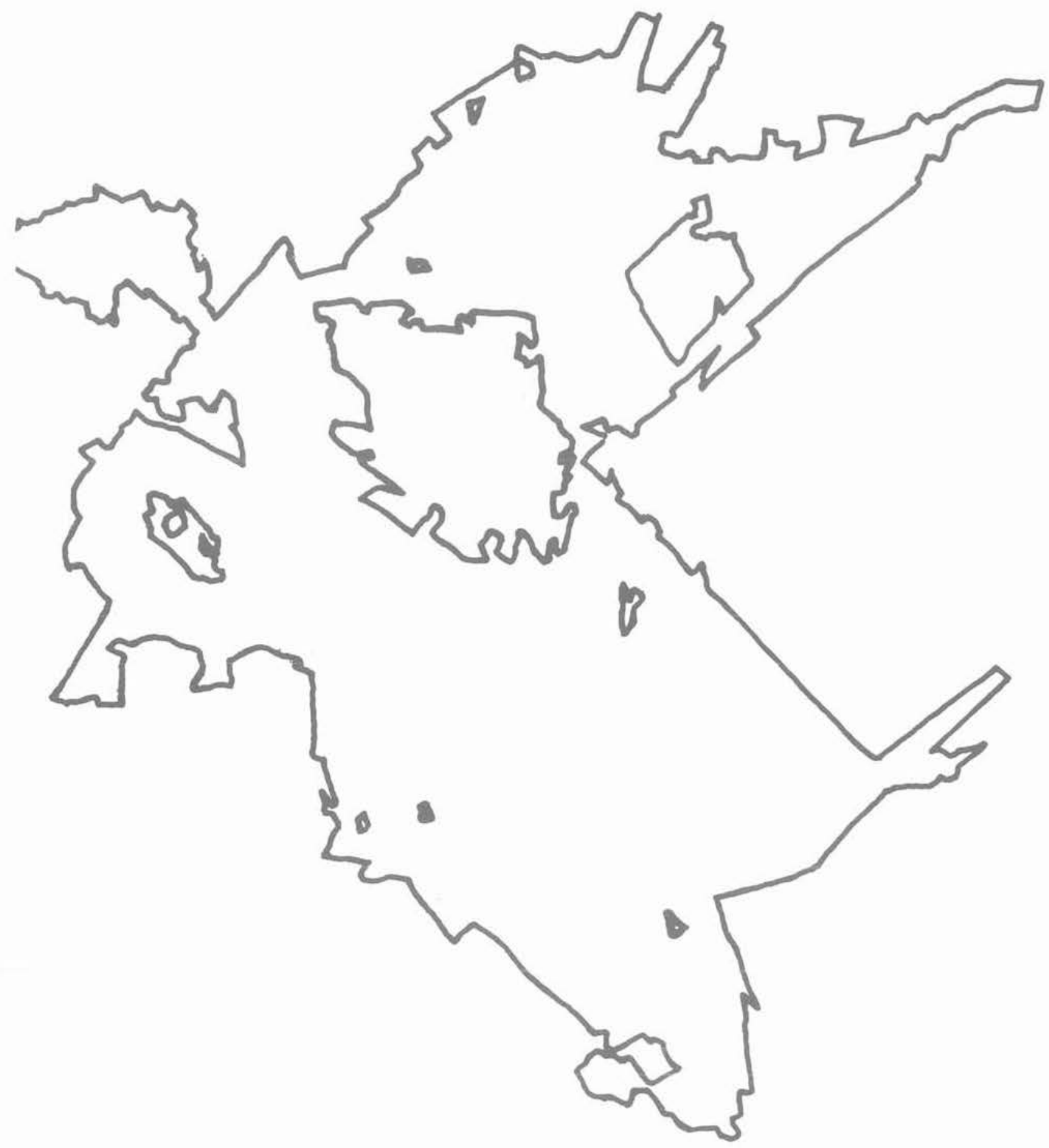

Fig. 3. - Frontière minéral-espace poreux - Lame mince - Calcaire dolomitique.

Fig. 3. - Mineral-pore space boundary - Thin section - Dolomitic limestone.

la ligne qui sépare, sur la coupe, le pore de son complémentaire (le solide minéral).

On mesure alors la longueur apparente $\mathrm{Li}$ de cette ligne pour différentes longueurs li de la toise utilisée: les résultats sont reportés sur un graphe $\mathrm{Log} \mathrm{Li}$ en fonction de Log li. Si l'objet est fractal de dimension fractale $D_{F}$, la relation entre ces deux quantités est linéaire, de pente $1-D_{F}$;
- pour une image présentant un ensemble de pores (fig. 2 et 4 ), on mesure la " quantité de matière " $M$ (surface couverte par les pores) contenue dans un champ d'observation de dimension $\mathrm{li}^{2}$ (par exemple: carré de côté li) ; les résultats sont reportés sur un graphe Log $\mathrm{M}$ en fonction de Log li. Si l'objet est fractal de dimension fractale $D_{F}\left(1 \leq D_{F} \leq 2\right)$, la relation entre ces deux quantités est linéaire de pente $D_{F}$. 


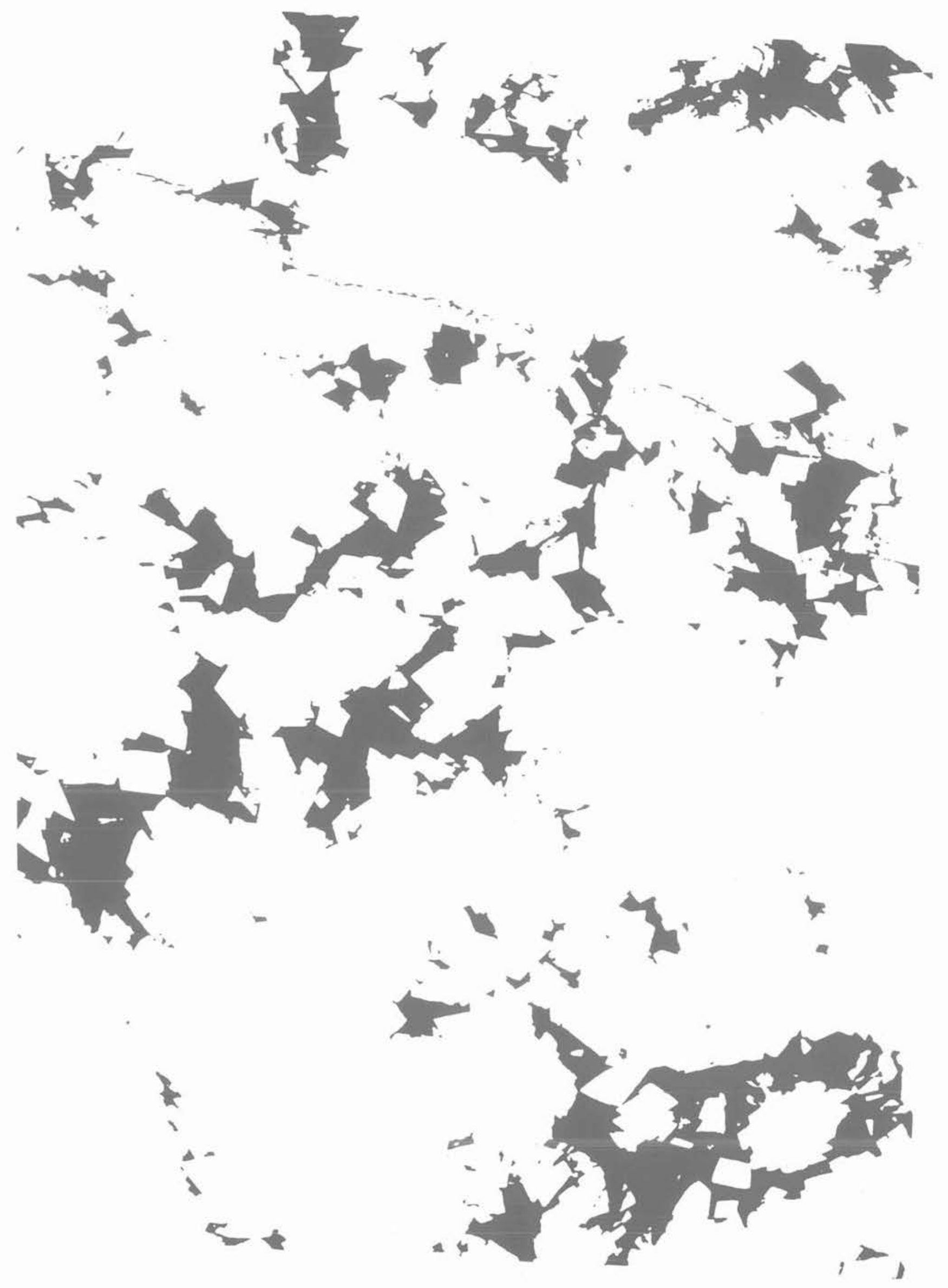

Fig. 4. - Lame mince. Calcaire dolomitique.

Fig. 4. - Thin section. Dolomitic limestone. 


\section{Résultats}

Les résultats sont reportés sur les figures 5 à 7 , qui appellent les commentaires suivants :

- les graphes obtenus montrent tous, pour une certaine gamme d'échelles de longueurs, une relation linéaire dont la pente permet à la fois de conclure à la nature fractale de l'objet étudié et de déterminer sa dimension fractale;

- pour certaines figures (5 et 7), le graphe indique, au-delà d'une valeur-seuil de l'échelle de longueur d'observation, une modification de comportement : on retrouve une relation linéaire, mais dont la pente, égale à deux, indique que l'objet examiné cesse d'être fractal.

\subsection{Domaine fractal et volume élémentaire représentatif}

L'approche classiquement adoptée pour décrire les phénomènes physiques qui se déroulent dans un matériau poreux consiste à raisonner sur un milieu continu fictif équivalent, homogène au moins localement.

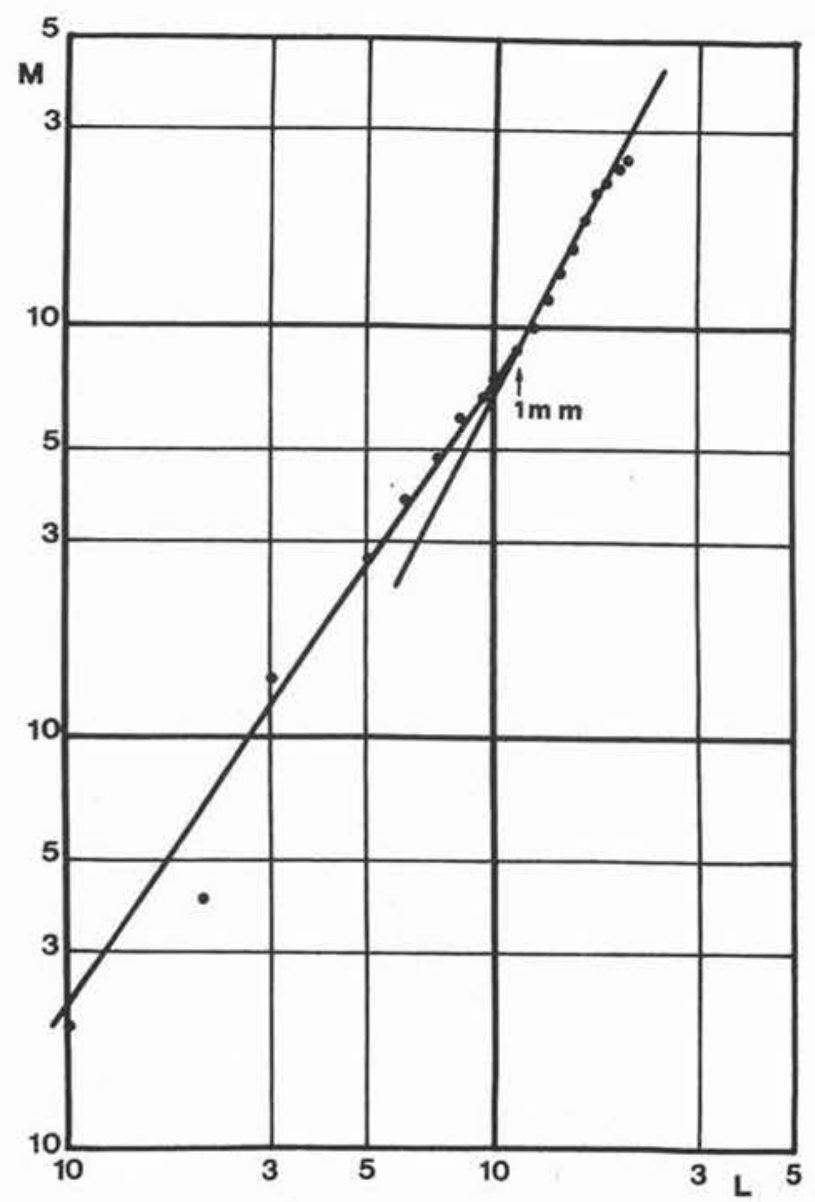

Fig. 5. - Analyse du réseau poreux. Grès de Fontainebleau (en noir sur la figure 2).

Fig. 5. - Analysis of the porous network Fontainebleau sandstone (black on figure 2).

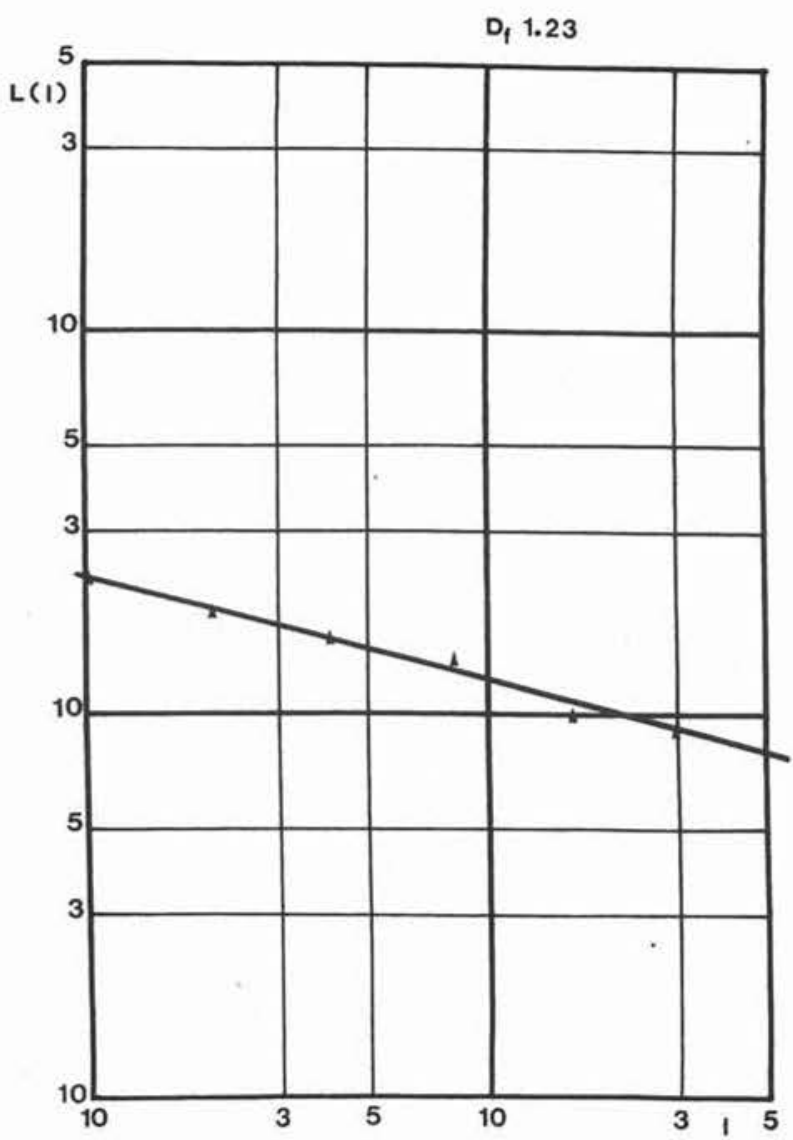

Fig. 6. - Analyse de la frontière minéral-espace poreux. Calcaire dolomitique (fig. 3).

Fig. 6. - Analysis of the mineral-pore space boundary. Dolomitic limestone (fig. 3).

Pour décrire par exemple le déplacement d'une phase fluide par une autre dans le matériau poreux, on considérera que, dans le milieu équivalent, chacune des phases fluides est présente en tout point.

On définit alors, en tout point, une valeur pour chacun des paramètres ou grandeurs physiques décrivant le système (porosité, perméabilité, vitesse et pression locale de chacun des fluides...). Cette valeur est considérée comme une moyenne (à définir, et d'ailleurs souvent assez mal définie...) des valeurs prises par la grandeur correspondante dans un volume, centré au point considéré, et qui soit à la fois :

- suffisamment étendu pour que l'opération de prise de moyennes ait un sens;

- suffisamment petit par rapport aux dimensions globales de l'échantillon, faute de quoi les variations des grandeurs moyennes ainsi définies resteraient trop faibles pour que l'on puisse espérer établir des lois macroscopiques utiles.

3.2.1. La notion de "Volume Elémentaire Représentatif " (voir § 1.2.) trouve à la fois sa justification, et une estimation de l'extension qu'il convient de lui donner, dans les exemples correspondant aux sections planes de réseaux poreux (lames minces) présentées sur les figures 2 et 4 . L'analyse de ces images montre en effet, comme on l'a indiqué précédemment, 


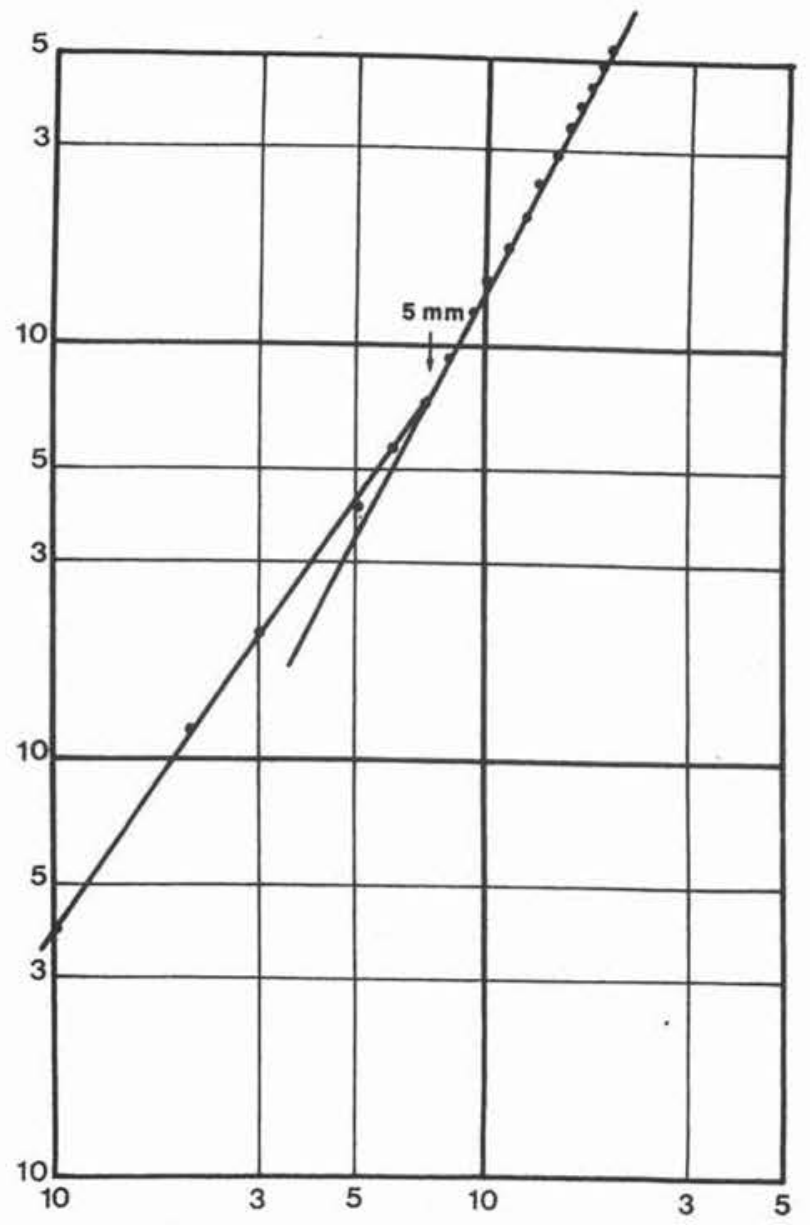

Fig. 7. - Analyse du réseau poreux. Calcaire dolomitique (en noir sur la figure 4).

Fig. 7. - Analysis on the porous network. Dolomitic limestone (black on figure 4).

que l'objet doit être considéré comme fractal en dessous d'une certaine échelle de longueur $l_{0}$, et comme de dimension entière au-delà (fig. 5 et 7 ).

Ce n'est qu'au-delà de $1_{0}$ que l'on peut définir une porosité qui ait une signification réelle; $1_{0}^{3}$ donne donc une estimation objective du volume élémentaire représentatif des matériaux considérés.

3.2.2. Il est instructif de noter les valeurs estimées de $1_{0}$ pour les deux roches poreuses analysées:

Grès de Fontainebleau : $1 \mathrm{~mm}$

Calcaire dolomitique : $5 \mathrm{~mm}$

Tout en restant prudent, et en gardant à l'esprit le fait qu'il ne s'agit là que d'ordres de grandeur, on notera que les différences sont largement significatives: $l_{0}$ peut être considéré comme une échelle caractéristique du réseau poreux du matériau (ou plutôt de l'échantillon).

3.2.3. Notons enfin que cette mesure du Volume Elémentaire Représentatif est celle qui correspond aux grandeurs liées directement - et uniquement - à la géométrie du matériau, soit, ici, la porosité.

\subsection{Le variogramme appliqué à l'analyse de la porosité}

\subsubsection{Exemple: la porositê du Grès de Fontainebleau}

a. L'analyse de la lame mince de Grès de Fontainebleau, dont la figure 4 fournit une photographie, a été présentée plus haut.

On a établi les conclusions résumées ici :

- le milieu est "fractal " à petite échelle ;

- le comportement devient "euclidien " pour des échelles d'observation supérieures au millimètre (soit de l'ordre de 4 diamètres de grains);

- ce comportement euclidien persiste au moins jusqu'aux échelles d'observation maximales que l'on a explorées, soit ici $10 \mathrm{~mm}$ environ.

b. On a cherché à compléter cette conclusion et à caractérister quantitativement la structure du milieu considéré sour l'aspect « porosité » - pour des échelles de longueurs supérieures, comprises entre le centimètre et le mètre [22 et 23].

Disposant de deux barreaux de Grès de Fontainebleau de dimensions $5 \mathrm{~cm} \times 5 \mathrm{~cm} \times 50 \mathrm{~cm}$, on a mesuré la porosité d'échantillons prélevés côte à côte dans ces barreaux, le pas d'échantillonnage étant de $2,5 \mathrm{~cm}$ (résultats sur la figure 8).

On a alors analysé ces mesures par la technique du variogramme.

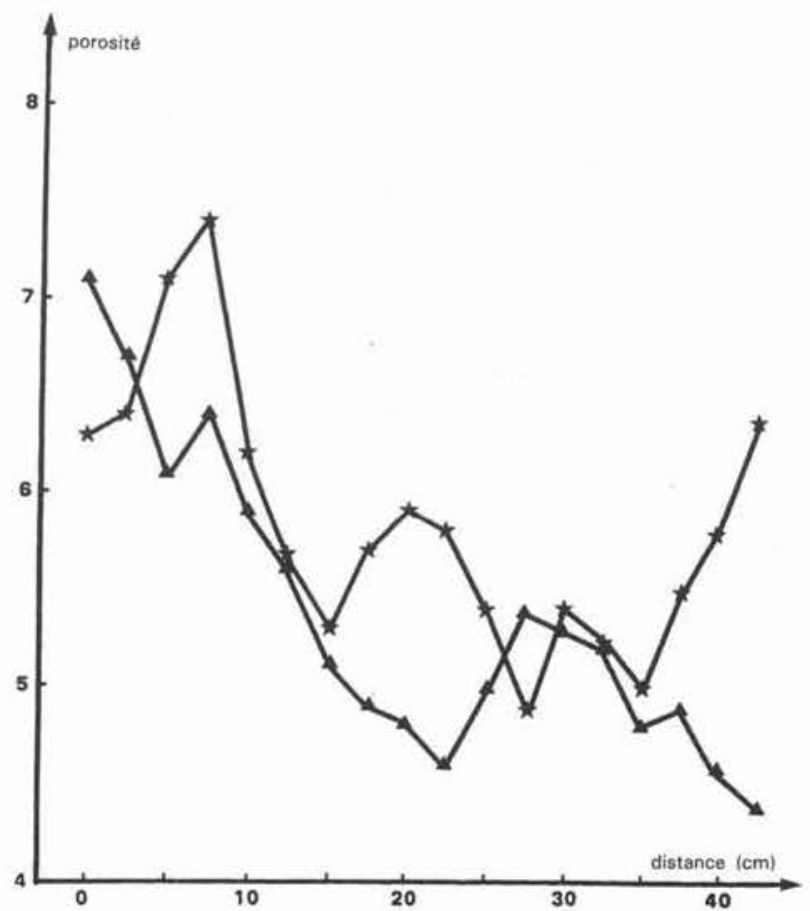

Fig. 8. - Variation de la porosité locale dans le Grès de Fontainebleau $(2$ barreaux de $50 \mathrm{~cm})$. Local porosity variations in Fontainebleau sandsone 12 rods, length $50 \mathrm{~cm})$. 
La figure 9 présente les résultats obtenus et conduit aux conclusions suivantes :

- l'allure du variogramme traduit l'existence de corrélations fortes entre les porosités mesurées sur des échantillons voisins (faibles valeurs du $\gamma(\mathrm{h})$, qui tend vers 0 quand $h$ tend vers 0 ), et la détérioration progressive de ces corrélations lorsque la distance $h$ qui sépare deux échantillons augmente ; le milieu présente donc une structure "hétérogène "(au sens de « non stationnaire ") aux échelles centimétriques :

- l'indication d'une tendance vers une asymptote quasi-horizontale pour les fortes valeurs de h traduit l'existence d'une "portée *-ou * échelle * - du phénomène * hétérogénéié de porosité *, estimée ici à $10-15 \mathrm{~cm}$, au-delà de laquelle le matériau peut être considéré comme globalement " homogène » (au sens de * stationnaire ) :

- au-delà de $25 \mathrm{~cm}$ environ, le variogramme expérimental perd ici toute signification, le nombre de couples de mesures associables devenant trop faible.

\subsubsection{Essai de synthèse sur l'exemple proposé}

a. Regroupons l'ensemble des informations acquises sur ce cas particulier de la porosité du Grès de Fontainebleau, en les classant par ordre d'échelles d'observation croissantes, et en extrapolant un peu vers les faibles dimensions :

- si l'on se situe très en dessous de la taille des grains (environ $250 \mu$ ), par exemple aux échelles inférieures à $10 \mu$. le champ d'observation implanté *au hasard » sur la lame mince de la figure 2 sera presque sûrement tout entier contenu soit dans les grains (" porosité „ nulle) soit dans les pores (" porosité " égale à $100 \%$ ) ; la réponse sera donc erratique et sans intérêt pratique ;

- pour des échelles de longueur comprises entre 0,1 et $1 \mathrm{~mm}$, le milieu possède une "logique interne "

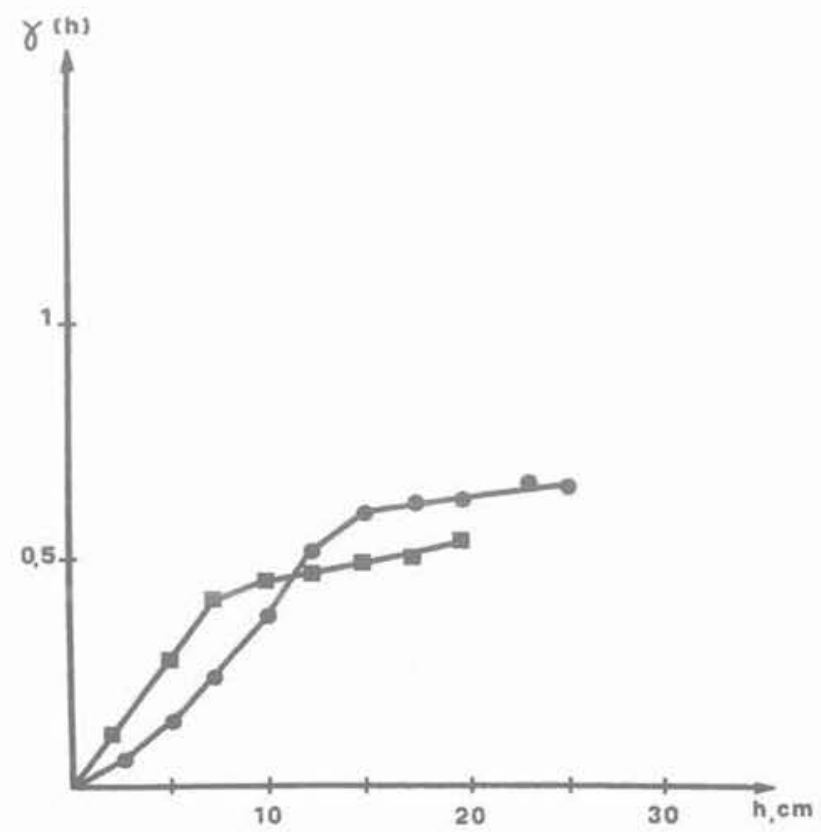

Fig. 9. - Variogrammes des porosités. Grès de Fontainebleau. Variogram of porosities. Fontainebleau sandstone. traduite par la structure fractale reconnue par la corrélation mise en évidence sur le graphe de la figure 5 (partie correspondant à une droite de pente inférieure à 2) ;

- aux échelles de longueur couvrant la gamme 1 $\mathrm{mm} \rightarrow 10 \mathrm{~mm}$, le matériau poreux présente une structure " euclidienne ", et il peut être modélisé en ce qui concerne la porosité - par un \& milieu homogène équivalent " pour lequel la notion de porosité à une signification et devient accessible à la mesure (fig. 5 , droite de pente 2) ;

- entre $25 \mathrm{~mm}$ et $15-20 \mathrm{~cm}$, on voit apparaître une nouvelle structuration du milieu, traduite par la partie ascendante du variogramme des porosités (fig. 9) ;

- au-delà de $15-20 \mathrm{~cm}$, le matériau perd à nouveau la « mémoire » de ses structures plus fines.

b. Une tentative d'illustration synthétique de ces conclusions est présentée sur la figure 10.

On notera essentiellement :

- la gamme très étendue des échelles de longueur explorées : de l'ordre de $100 \mu$ à $1 \mathrm{~m}$, soit dans un rapport de 1 à $10^{4}$ (dont il faut soustraire une tranche étroite, couvrant les échelles de $1 \mathrm{~cm}-2 \mathrm{~cm}$ environ ; cette lacune, résultant du manque de données disponibles, paraît sans incidence sur les conclusions énoncées ici),

- la succession de domaines alternativement « irréguliers " et "réguliers ", que l'on peut considérer comme traduisant l'existence de structures emboîtées les unes dans les autres, à rapprocher des " structures gigognes " mises en évidence par SERRA [25], et dont les échelles se répartissent fréquemment selon une progression géométrique (noter l'échelle logarithmique adoptée pour présenter les résultats regroupés sur la figure 10).

\subsection{Extension \\ à d'autres "grandeurs moyennes»}

L'analyse de la répartition de l'espace poreux du Grès de Fontainebleau a permis de préciser la notion de Volume Elémentaire Représentatif et de reconnaître les limites dans lesquelles cette notion est applicable.

On se gardera toutefois de considérer que le Volume Elémentaire Représentatif ainsi défini, dans les gammes d'échelles de longueur où il correspond à une réalité physique, reste "représentatif " lorsque l'on s'intéresse à une autre grandeur pétrophysique. Deux exemples sont instructifs à cet égard.

\subsubsection{Structure des perméabilités du Grès de Fontainebleau}

La figure 11 présente un variogramme des perméabilités du Grès de Fontainebleau; elle met en évidence [39]:

- une structure en « ilots 》 de tailles centimétriques (variogramme présentant un a effet de trou ») ;

- une portée, à plus grande échelle, de l'ordre de 30 à $40 \mathrm{~cm}$. 


\section{GRES DE FONTAINEBLEAU}

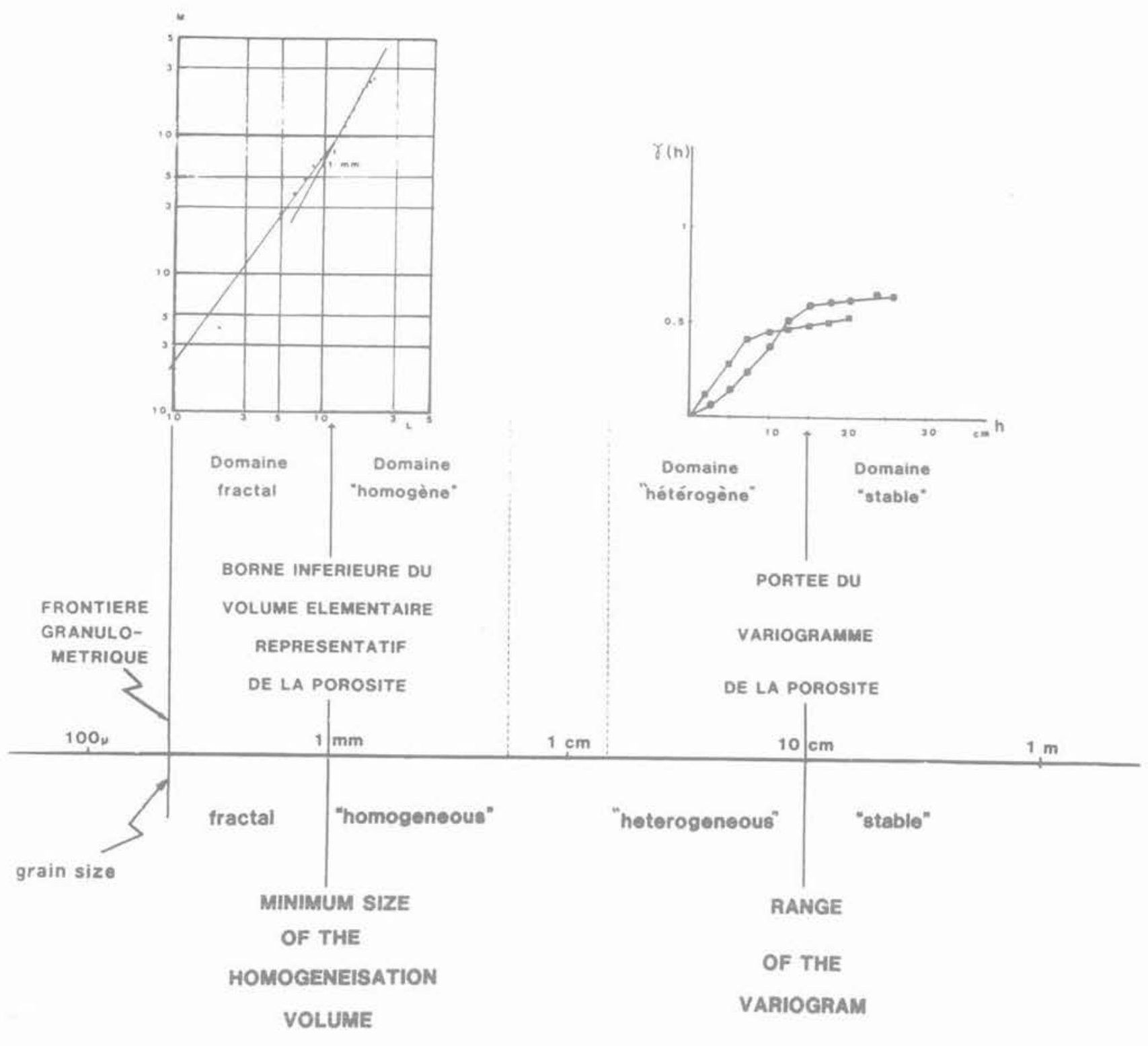

FONTAINEBLEAU SANDSTONE

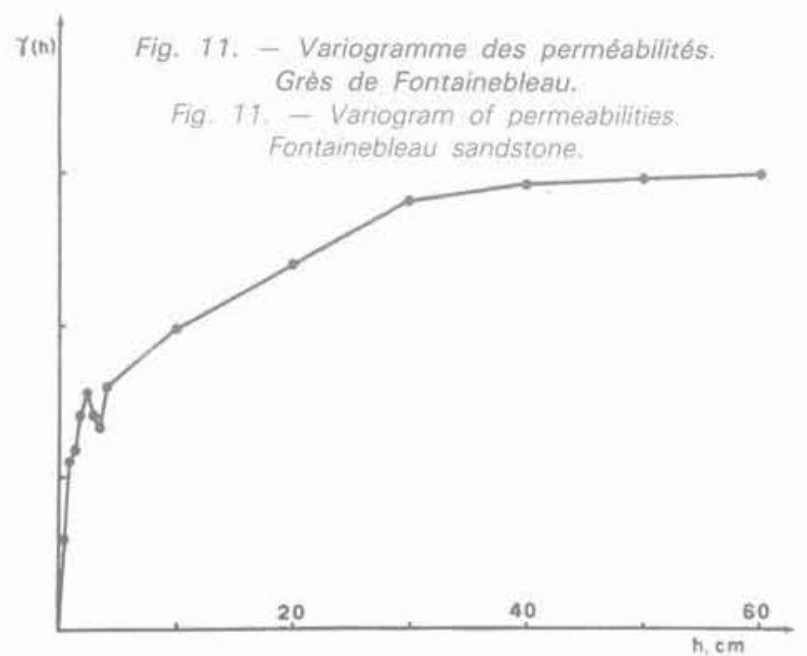

Fig. 10. - Structure de la porosité. Grés de Fontainebleau. Fig. 10. - Structure of the porosity. Fontainebleau sandstone

Les échelles caractéristiques ainsi estimées sont significativement distinctes de celles observées pour la porosité.

3.4.2. Répartition des fluides lors d'une expérience de déplacement

On a montré (voir fig. 12) que l'interface liquide-gaz observé lors du drainage par gravité d'une colonne poreuse (de structure a priori simple, voisine de celle du Grès de Fontainebleau) est fractal, au moins aux échelles d'observation inférieures au diamètre de la colonne, soit $5 \mathrm{~cm}$ ([1] et [2]) ; le Volume Elémentaire Représentatif - s'il existe - doit donc avoir ici 


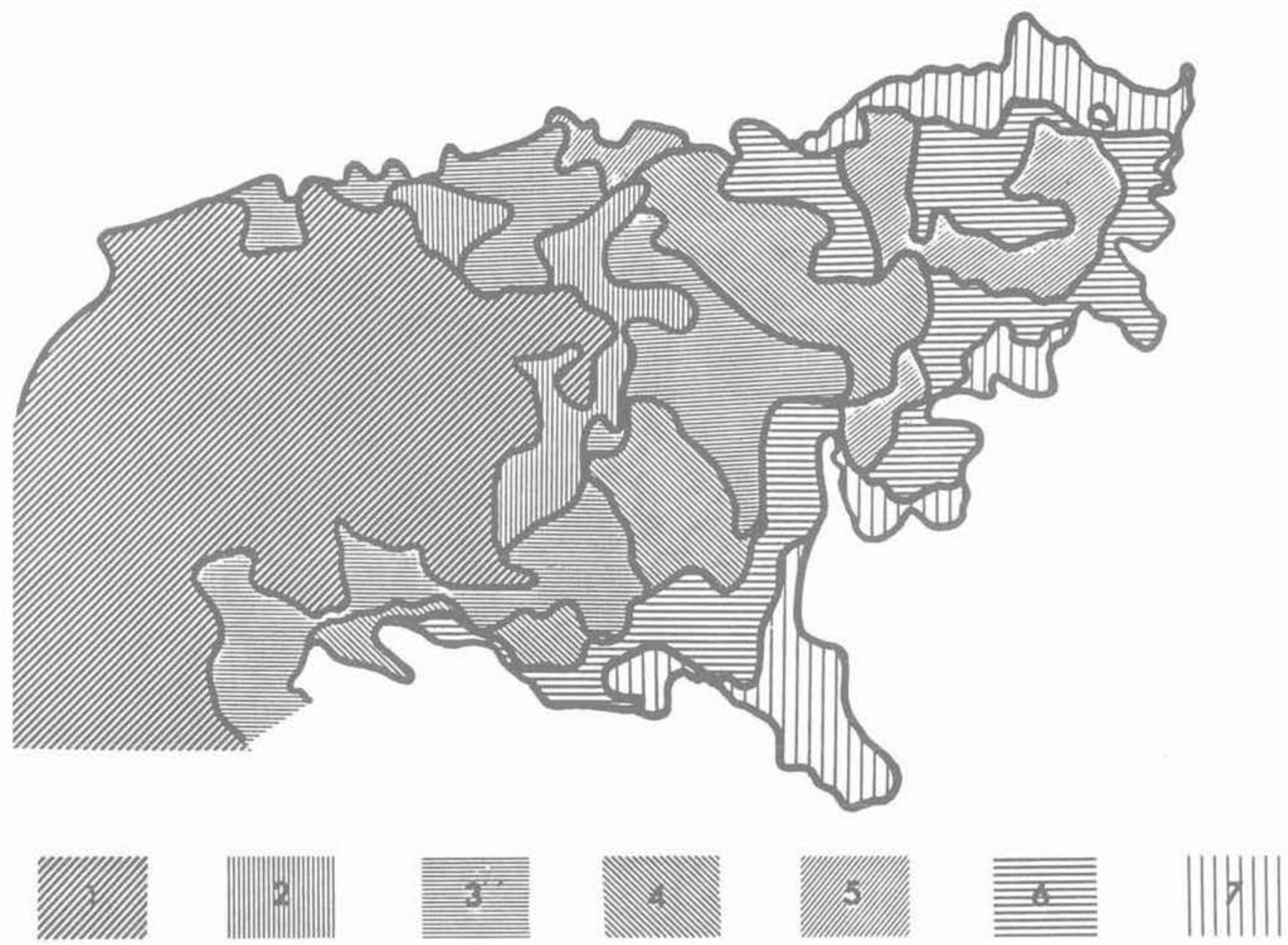

Fig. 12. - Interface gaz-liquide dans une colonne poreuse (Sept étapes successives). Fig. 12. - Gas-liquid interface in a porous column (Seven successive steps).

une extension qui déborde les dimensions de l'échantillon et, en conséquence, la notion de * saturation * est ici, à strictement parler, inutilisable, car sans signification.

\section{CONCLUSIONS}

\subsection{Bilan global actuel}

On résumera comme suit les résultats acquis :

a. On dispose de techniques qui peuvent apporter une contribution utile à l'étude et à la description des objets naturels, et en particulier des structures complexes des matériaux géologiques.

b. Certains phénomènes physiques (par exemple: les déplacements de fluides) dont ces matériaux sont le siège peuvent engendrer eux-mêmes des structures complexes éventuellement fractales (fronts de déplacements diphasiques, [1] et [2]) ou ne plus obéir aux lois classiquement admises (exemple: diffusion qui n'est plus décrite par une loi en racine carrée du temps). c. Les particularités que l'on traduit depuis quelques années par la notion d'objet fractal sont étroitement liées avec celles qui avaient déjà été reconnues depuis longtemps par certains praticiens, et que la géostatistique a permis, depuis environ trente ans, de prendre en compte, à la fois sur le plan théorique et au niveau des applications industrielles.

\subsection{Résultats pratiques acquis}

On retiendra principalement les points suivants :

4.2.1. Notion de «Volume Elémentaire Représentatif

Un premier résultat particulièrement important paraît être la mise en évidence objective d'échelles de longueur caractéristiques.

On notera que cette situation, si elle se présente fréquemment, ne recouvre sans doute pas la totalité des cas possibles (voir plus loin).

Dans les cas où de telles frontières existent, la notion de *Volume Elémentaire Représentatif * possède une signification objective, et on peut accéder à une estimation de sa mesure (pour une grandeur ou pour un phénomène physique donné). 


\subsubsection{Structures emboîtées (ou gigognes)}

Si la gamme des échelles de longueur accessible à l'exploitation est suffisamment large, il arrive que l'analyse amène à mettre en évidence plusieurs transitions correspondant à autant de structures, identifiables par leurs échelles caractéristiques croissantes, et contenues les unes dans les autres.

Cette notion de structures emboitées ou gigognes (pour reprendre des expressions déjà utilisées par MATHERON [32] et par SERRA [25]) est bien illustrée par l'exemple du Grès de Fontainebleau.

\subsubsection{Limites de validité de la notion de « Volume Elémentaire Représentatif »}

On peut concevoir des situations où, dans toute la gamme des échelles auxquelles un phénomène se déroule, il n'apparaisse aucune plage où la notion de Volume Elémentaire Représentatif ne soit applicable.

Ceci peut se réaliser :

- soit parce que la taille du domaine observable est trop réduite pour que lon atteigne un état stationnaire :

- soit parce qu'il existe des structures emboîtées qui se recouvrent mutuellement, aucune d'entre elles ne parvenant à se développer suffisamment (pour que le milieu devienne stable) avant que la suivante n'apparaisse.

Cette situation est bien illustrée par le schéma théorique proposé par SERRA [25] et qui montre qu'elle se traduit par exemple par des variogrammes de type De WIJS $(\gamma(h)=\alpha \cdot \log h)$.

\subsubsection{Représentativité des mesures physiques sur échantillons}

La notion de «Volume Elémentaire Représentatif " ne possède donc pas de valeur absolue, applicable à l'ensemble des propriétés macroscopiques décrivant le comportement d'un matériau. On peut dès lors s'interroger sur la signification réelle de certains types d'essais réalisés sur échantillons et sur la valeur des grandeurs physiques déduites de ces essais, qui sont habituellement interprétés en supposant que les échantillons sont représentatifs pour l'ensemble des grandeurs macroscopiques correspondantes (par exemple: porosité, perméabilité, saturation en fluides, pour une expérience de déplacement).

\subsection{Voies de recherches à explorer}

\subsubsection{L'aspect description géométrique mérite d'être étendu :}

a. au niveau des méthodes, tout d'abord : des progrès touchant à la mécanisation - sinon l'automatisation au moins partielle - des procédés d'analyse géométrique paraissent souhaitables ;

b. au niveau des matériaux ensuite: on a testé l'intérêt de lapproche proposée en choisissant des objets géologiques simples. L'exploration de matériaux pré- sentant des structures plus complexes paraît souhaitable.

En ce qui concerne l'aspect méthodologique, on signalera deux approches complémentaires qui font l'objet de développement actuels.

c. L'étude de la structure des matériaux rocheux par scanner $\mathrm{X}$.

Cette technique, d'utilisation courante dans les domaines médicaux, permet l'exploration détaillée d'échantillons massifs, et présente l'avantage d'être non destructrice. Son utilisation permet donc, outre l'étude des structures internes d'échantillons, de sélectionner ceux qui seraient "représentatifs », donc à retenir pour une expérimentation en laboratoire.

Une illustration de cette technique est présentée sur la planche photo de la figure 13 ; l'image correspond à une tranche d'épaisseur millimétrique explorée directement à l'intérieur de l'échantillon.

Les différents niveaux de gris sont corrélés à la masse volumique locale du matériau.

d. L'utilisation d'équipements et de logiciels de traitement et d'analyse pour des images de ce type (ou d'images analogues obtenues d'après lames minces) répond au souci d'automatisation des procédures d'étude, évoqué plus haut.

La figure 14 présente le résultat obtenu en transformant, par seuillage et discrétisation, une image scanner, initialement en niveaux de gris, en image noir et blanc.

La figure 15 illustre l'efficacité de certains algorithmes basés sur les méthodes de morphologie mathématique; on a cherché à ne retenir, de l'image scanner initiale, que les structures majeures, plus ou moins linéaires, qui recoupent le document (échantillon de craie).

4.3.2. La modélisation géométrique des matériaux poreux doit être abordée sous un angle permettant de prendre en compte les résultats acquis et de restituer au mieux la complexité des milieux réels.

A titre d'illustration de ceci, on citera deux domaines d'application possibles :

a. L'écoulement des fluides dans les roches-réservoirs :

P.M. ADLER [10] [11] [12] [13] [14] a abordé les problèmes de transport dans des structures périodiques dont les cellules élémentaires sont construites par des processus itératifs simples qui engendrent des géométries fractales.

Parmi les résultats obtenus, on retiendra notamment l'incidence, sur la valeur de la perméabilité, des particularités géométriques des différentes structures proposées.

Au-delà de cette première approche, des travaux récents [41] montrent qu'il est possible d'estimer la 


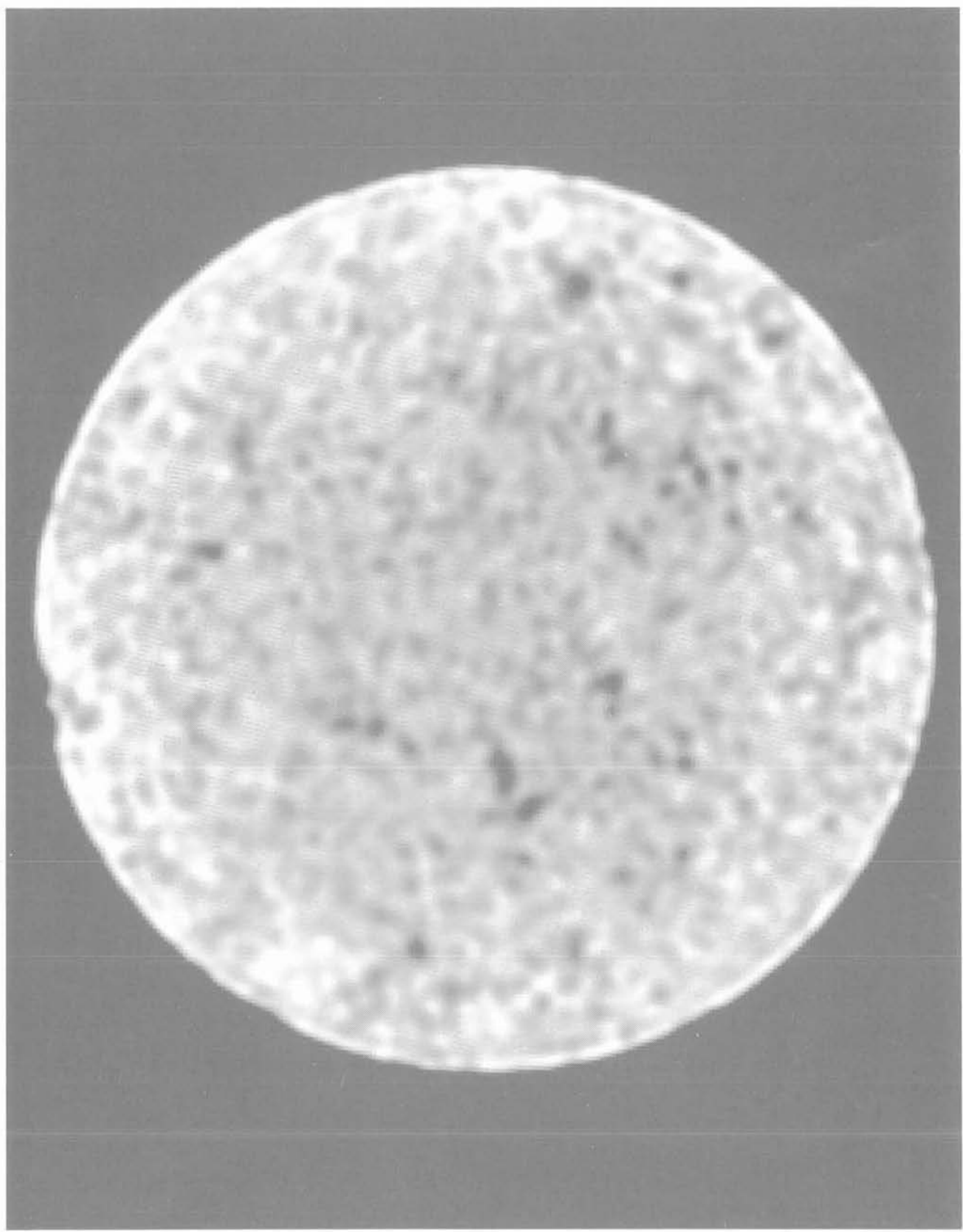

Fig. 13. - Image scanner X. Calcaire.

Fiq. 13. - X ray scanner analysis. Limestone. 


\section{X-RAY SCANNER ANALYSIS}

\section{LIMESTONE}
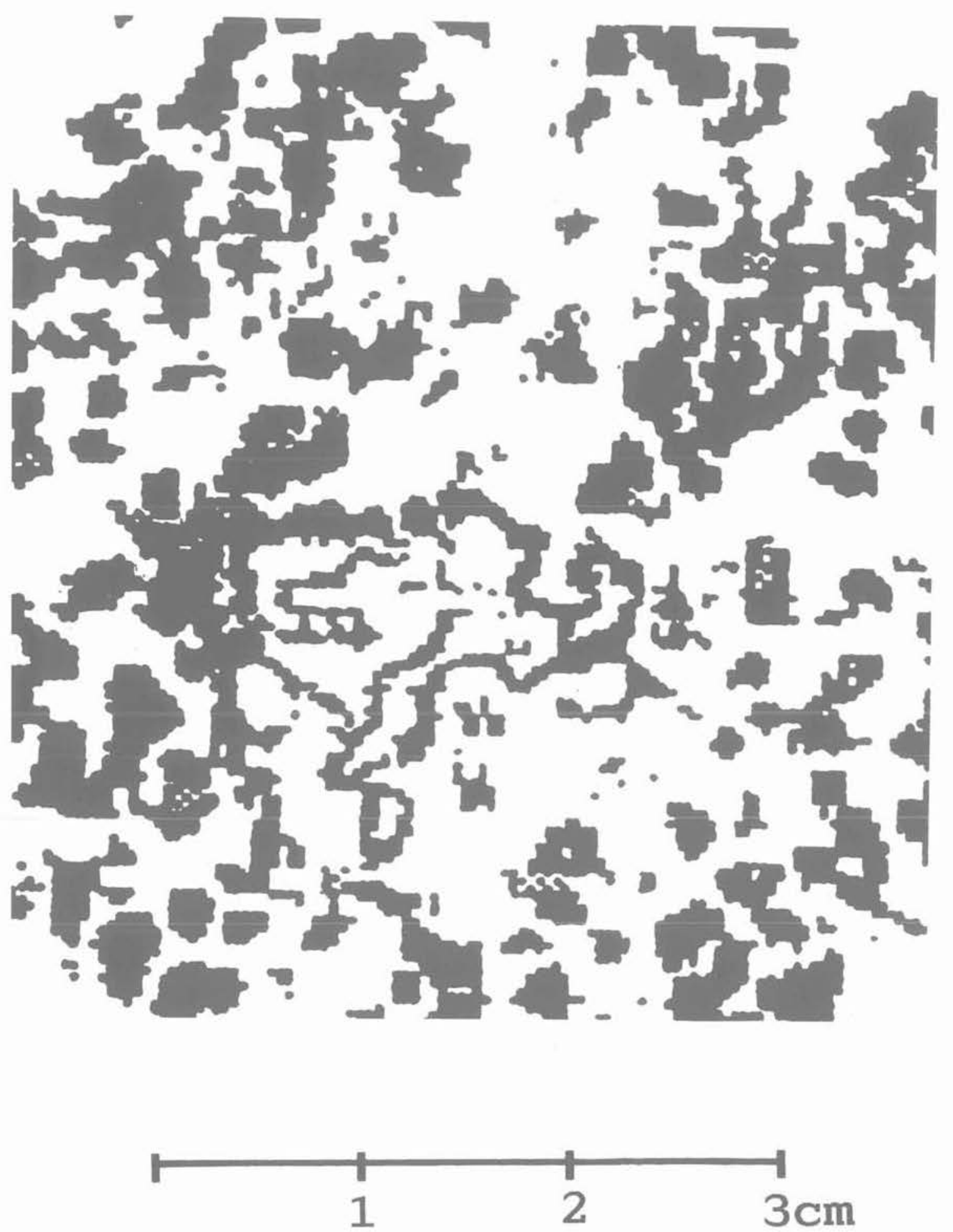

Fig. 14. - Image seuillée et digitalisée.

Fig. 14. - Thresholding and digitalization. 

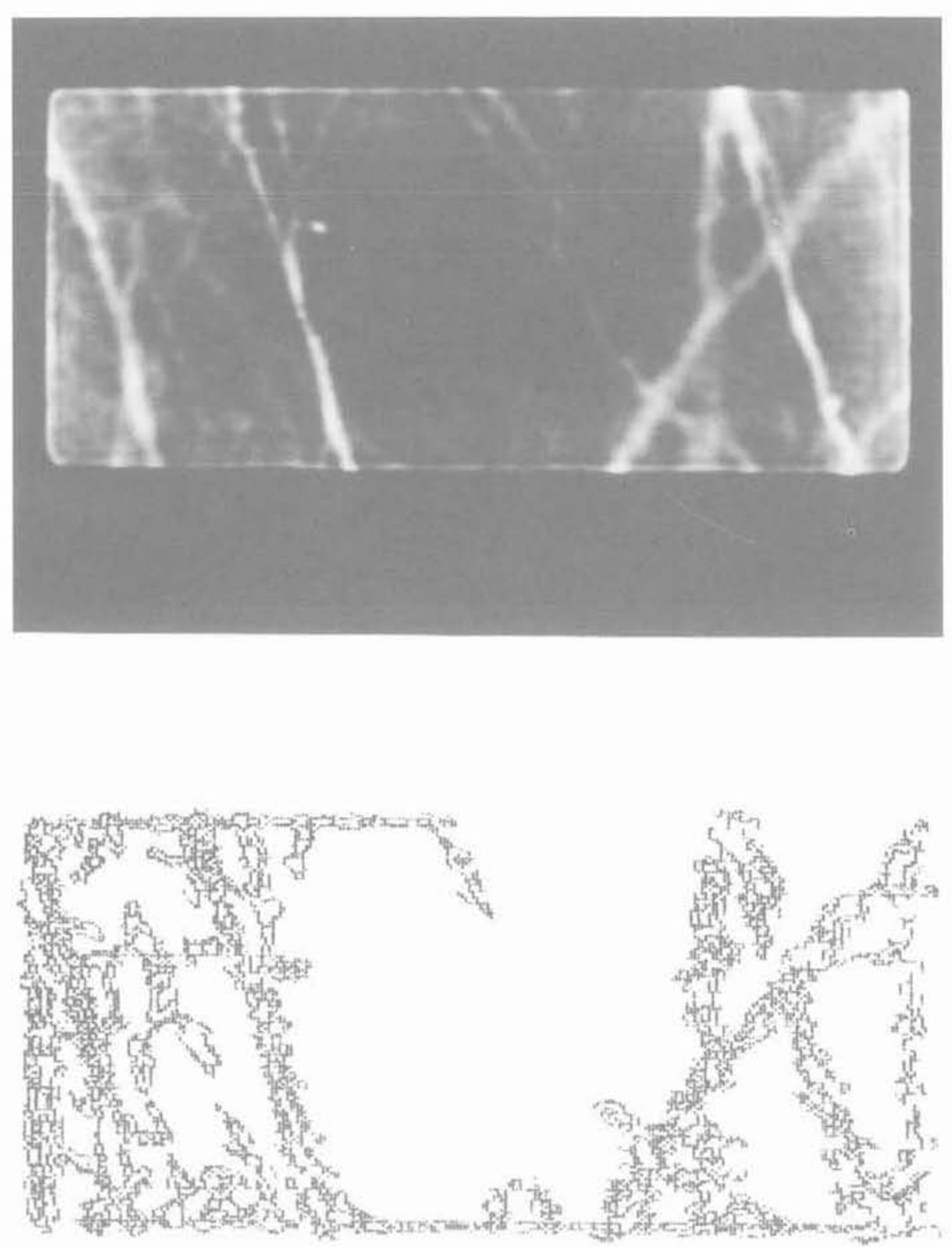

Fig. 15. - Analyse par scanner X. Echantillon de craie. Fig. $15-X$ ray scanner analysis Chalk sample.

perméabilité d'un matériau naturel à structure granulaire (Grès de Fontainebleau par exemple), à partir de données mesurées directement sur lame mince: porosité et fonction d'autocorrélation de l'espace des pores (on notera que le comportement à l'origine de la fonction d'autocorrélation traduit le degré de régularité - et éventuellement le caractère fractal - du matériau).

b. La propagation d'un signal (mécanique ou électromagnétique) :

On dispose de quelques résultats [42] qui montrent l'effet des interactions ondes-structures, en fonction notamment des rapports $\frac{\lambda}{\mathrm{Li}}$ entre la longueur d'onde $\lambda$ et les échelles de longueur $\mathrm{Li}$ - éventuellement multiples - qui caractérisent les structures du matériau.
L'étude approfondie de ces interactions devrait constituer un apport bénéfique pour les techniques, basées sur des phénomènes de propagation d'ondes, qui ont pour objet la caractérisation d'un milieu naturel (exemples: sismique, diagraphies).

\section{BIBLIOGRAPHIE}

[1] JACQUIN Ch. (1984), Note sur le caractère fractal des interfaces fluide-fluide en milieu poreux. Rapport IFP Réf. 32 341, Juillet 1984.

[2] JACQUIN Ch. (1985), Caractère fractal des interfaces fluide-fluide en milieu poreux. Comp. tes rendus à l'Académie des Sciences. Paris, Série 2 , Tome $300, n^{\circ} 15$.

[3] JACQUIN Ch., ADLER P.M. (1983), The frac. tal dimension of a gas-liquid interface in a porous 
medium. Journal of Colloid and Interface Science, Vol. 107, n ${ }^{\circ}$, October 1983, p. 405-417.

[4] AVNIR D., FARIN D., PFEIFER P. (1984), Molecular fractal surfaces. Nature, Vol. 308, 15 mars 1984 , p. 261-263.

[5] BALE H.D., SMITH P.W. (1984), Small angle $X$ ray scattering investigation of submicroscopic porosity with fractal properties. Physical Review Letters, Vol. $53, n^{\circ} 6,6$ aout 1984 p. 596-599.

[6] KATZ A.J., THOMPSON A.H. (1985), Fractal sandstone pores: implication for conductivity and pore formation. Physical Review Letters, Vol. 54, n० 12, 25 mars 1985, p. 1325-1328.

[7] JACQUIN Ch. (1985), Caractère fractal des réseaux de discontinuité des massifs rocheux. Rapport IFP. Réf. 33 699, Décembre 1985.

[8] SCHOLZ C.H., AVILES C. (1985), Fractal dimension of the 1906 San Adreas Fault and 1915 Pleasant Valley Faults. Annual Meeting of the Seismological Society of America, 14-16 avril 1985, Austin, Texas.

[ 8 bis] AVILES C.A., SCHOLZ C.H., BOAT WRIGHT J., Fractal analysis applied to characteristic segments of the San Andreas Fault. J. Geophys. Res., Vol. 92, n B1, 10 janv. 1987. pp. 331-344.

[9] BENENATI R.F., BROSILOW C.B., (1962), Void fraction distribution in beds of spheres. A.I.C.H.E. Journal, Vol. 5, n 3, July 1962 , p. 359-361.

[10] ADLER P.M. (1985). Transport process in fractals I - Conduction and permeability of a Leibniz packing in the lubrication limit. Int. J. Multiphase Flow, Vol. 11, n० 1, p. 91-108.

[11] ADL.ER P.M. (1985), Transport process in fractals II - Stokes flow in fractal capillary networks. Int. J. Multiphase Flow, Vol. 11, n०2, pp. 213 239.

[12] ADLER P.M. (1986), Transport processes in fractals VI - Stokes flow in Sierpinski carpets. Phy. Fluids, 29, p. 15

[13] ADLER P.M., Perméabilité d'un milieu poreux aléatoire bidimensionnel. Comptes rendus Acad. Sciences, Paris.

[14] ADLER P.M., JACQUIN C.G. (1987), Fractal porous media. I Stokes flow in random carpets. Transport in Porous Media, 2, p. 553-569.

[15] JACQUIN G., ADLER P.M. (1987), Fractal porous media - II - Geometry of porous geological structures. Transport in Porous Media, 2, p. 571-596.

[16] MANDELBROT B. (1975), Les objets fractals : forme, hasard et dimension. Flammarion, Paris.

[17] MANDELBROT B. (1975), Hasard et tourbillons. Quatre contes à clefs. Annales des Mines, Nov. 1975 , p. 61-66.
[18] DE WIJS H.J. (1951, 1953), Statistics of ore distribution. Geologie in Mijnbouw, 13 (1951). p. $365-375$ et 15 (1953), p. 12-24.

[19] FELLER W. (1966), Introduction to probability theory and its applications. John Wiley and sons.

[20] MATHERON G. (1967), Kriging or polynomial interpolation procedures? The canadian mining and metallurgical bulletin. Transactions, vol. LXX, p. 240-244.

[21] MATHERON G. (1965), Les Variables régionalisées et leur estimation. Masson, Paris,

[22] SAVOLDELLI P. (1984), Etude hydraulique et acoustique des hétérogénéités du Grès de Fontainebleau. Rapport de stage. Rapport IFP, Réf. 32 318, Juillet 1984 .

[23] JACQUIN Ch., LEGAIT B. (1985), Approche probabiliste des milieux hétérogènes ou fractu. rés, en relation avec les écoulements diphasi. ques. Symposium AlRH, Montvillargenne, 3-7 juin 1985.

[24] SERRA J. (1982), Image analysis and mathematical morphology. Academic Press, Chapitre $\mathrm{V}, \mathrm{D} 4$ * The fractal behaviour of clay material , p. $152-158$

[24 bis] KOLOMENSKI E.N., SERRA J. (1976), Trois études de morphologie mathématique en géologie de l'ingénieur. Bulletin de l'Association Internation de Géologie de l'Ingénieur, $n^{\circ} 13$, p. 89-97.

[25] SERRA J. (1965), Les structures gigognes: morphologie mathématique et interprétation métallogénique. Mineralium Deposita (Berl.). 3 p. 135-154.

[26] DANIS M., JACQUIN Ch. (1983), Influence du contraste des viscosités sur les perméabilités rela. tives lors du drainage. Expérimentation et modélisation. Revue de l'IFP, Vol. XXXVIII, $n^{\circ} 6$, Nov-Déc. 1983, p. 723-734.

[27] JACQUIN Ch. (1964), Relations entre la perméabilité et les caractéristiques géométriques du Grès de Fontainebleau. Revue de IIFP, Vol XIX, Juillet-Août 1964, p. 921-937.

[28] GUILLOT D. (1982), Propriétés physiques et texturales d'un modèle artificiel d'un matériau à porosité intergranulaire. Thèse Paris VI. Ecole des Mines, 14 juin 1982.

[29] CONRAD F., JACQUIN Ch. (1973), Représentation d'un réseau bidimensionnel de failles par un modèle probabiliste. Application au calcul des grandeurs géométriques des blocs matriciels: Revue de l'IFP, Vol. XXVIII, nº 6, Nov-Déc. 1973 , p. $843-890$.

[30] MAHE M.J., ADLER P.M., JACQUIN Ch. (1985), La dimension fractale d'un interface gaz. liquide dans un milieu poreux. Symposium international sur l'approche stochastique des écoulements souterrains. AIRH, Montvillargenne, 3-7 juin 1985.

[31] MATHERON G., DE MARSILY G. (1980), Is transport in porous media always diffusive? A counter example. Water resources research, 16 (4). 
[32] MATHERON G. (1987), Eléments pour une théorie des milieux poreux. Masson, Paris.

[33] REDNER S., KOPLIK J., WILKINSON D., Hydrodynamic dispersion in a self-similar geometry (preprint).

[34] ALLAIN C., CLOITRE M, (1985), Optical Fourier transforms of fractals « fractals in physics ». Trieste, Juillet 1985.

[35] OJO S.B., MEREU R.F. (1986), The effect of random velocity functions in the travel times and amplitudes of seismic waves. Geophys. J. R. ast. Soc., 1986, Vol. 84, p. 607-618.

[36] JAKEMAN E., HOENDERS B.J. (1982), Scattering by a random surface of rectangular grooves.Optica Acta, Vol. 29, n 12, p. 1587-1598.

[37] BOURBIAUX B. (1986), Rapport de synthèse sur la mise à l'échelle des essais d'imbibition sur des milieux poreux parfaitement mouillés par l'eau. Rapport IFP-ARTEP, Réf. IFP 34053 , Avril 1986.
[38] MATHERON G. (1965), Les variables régionalisées et leur estimation. Masson, Paris.

[39] JACQUIN Ch. G., ADLER P.M. (1987), Structure of porous networks and fluid flow in rock masses. Paper presented at the International Workshop on Forced fluid flow through strong fractured rock masses. Garchy (France) 13-15 April 1987. Commission of the European Committees EUR 11164/2.

[40] JACQUIN Ch. G., ADLER P.M. (1987), Fractal geological structures. Paper presented at the 7 th International Congress for stereology, Caen, 2-9 Sept 1987. Acta Stereologica, 1987; 6/III, 821-826.

[41] ADLER P.M., JACQUIN Ch. G., QUIBLIER J.A. (1989), Flow in simulated porous media. Soumis à publication.

[42] LAFAIT J., TANNER B.D. (Editors) (1988), ETOPIM 2. Proceedings of the Second international conference on electrical transport and optical properties of inhomogeneous media. 29 August-2 September 1988, Paris, France, NorthHolland, Amsterdam, 1989. 